\title{
Auditory brainstem responses in a Rhesus Macaque model of neuro-AIDS
}

\author{
Leigh AM Raymond ${ }^{1,2}$, Dennis Wallace ${ }^{2}$, Nancy EJ Berman ${ }^{2,5}$, Joanne Marcario ${ }^{1,2}$, Larry Foresman ${ }^{4}$, \\ Sanjoy V Joag ${ }^{3}$, Ravi Raghavan ${ }^{3}$, Opendra Narayan ${ }^{2,3}$, Paul D Cheney ${ }^{1.2}$ \\ Departments of ${ }^{1}$ Molecular \& Integrative Physiology, ${ }^{2}$ Smith Mental Retardation and Human Development Research \\ Centre, ${ }^{3}$ Marion Merrell Dow Laboratory For Viral Pathogenesis, ${ }^{4}$ Laboratory Animal Resources, ${ }^{5}$ Anatomy and Cell \\ Biology, University of Kansas Medical Center, 3901 Rainbow Blvd., Kansas City, Kansas 66160, USA
}

\begin{abstract}
Nine rhesus macaques (Macaca mulatta) were inoculated with a combination of two passaged strains of $\mathrm{SIV}_{\text {mac }}$ (R71 and 17E), both of which are known to be neurovirulent. Auditory brainstem responses (ABRs) were recorded at regular intervals from these animals both before and after inoculation. Increases in ABR peak and interpeak latency were observed corresponding to progression of SIV disease. Post-inoculation increases in latency were observed for all five peaks of the ABR and for interpeak intervals I-V and III-V. The largest increases in latency were associated with end-stage disease. Within 14 weeks of inoculation, all but two animals developed end-stage simian AIDS and were euthanized. Histopathological examination revealed multifocal lesions in the cerebral gray and white matter as well as in the auditory structures of the brainstem. In most animals, ABR changes were accompanied by evidence of underlying neuropathology. However, cases of severe neuropathology with no ABR abnormalities and vice versa were also noted. Though in a much shorter time frame, $\mathrm{SIV}_{\text {mac }} \mathrm{R} 71 / 17 \mathrm{E}$ produced both physiological and histopathological abnormalities similar to those associated with HIV disease in humans. These results further support the $\mathrm{SIV}_{\text {mac }} \mathrm{R} 71 / 17 \mathrm{E}$ infected rhesus macaque as an animal model of HIV related neurological disease in humans.
\end{abstract}

Keywords: monkey; evoked potentials; ABR; SIV; HIV; pathology

\section{Introduction}

It is well established that HIV not only causes loss of immune competence but also infects the central nervous system (CNS). Among the manifestations of HIV infection is the occurrence of severe neurological disease including neuropathy, myelopathy and impairments in memory, intellectual ability, visuospatial performance and motor function (Navia et al, 1986a; Navia and Price, 1987; Perry and Marotta, 1987; Price et al, 1988; Janssen et al, 1989; Butters et al, 1990; Krikorian and Wrobel, 1991; Stern, 1991; Egan 1992; Arendt et al, 1992; Bankaitis and Keith, 1995). During the later stages of HIV disease (CDC stage IV), cognitive and motor impairments can be easily demonstrated with standard clinical neuropsychological testing. This constellation of symptoms is a common feature of AIDS and has been referred to as AIDS related dementia. The existence of early cognitive and motor deficits

Correspondence: PD Cheney

Received 14 November 1997; revised 20 June 1998; accepted 22 June 1998 associated with the asymptomatic phase of HIV infection is now also clear, although these deficits may be quite subtle (Borstein et al, 1992; Sinforiani et al, 1991; Clifford et al, 1990; McAllister et al, 1992; Perry et al, 1989; Riedel et al, 1992; Arendt et al, 1990; Martin et al, 1991, 1992a, b; Karlsen et al, 1992a, b). Upon post mortem examination, estimates of the number of AIDS patients with CNS lesions range as high as $80 \%$ (Elder et al, 1988).

The physiological integrity of the auditory system in symptomatic and asymptomatic HIV-infected human subjects has been widely investigated using auditory brainstem evoked responses (Rosenhall et al, 1989; Hausler et al, 1991; Birchall et al, 1992; Pagano et al, 1992; Bankaitis, 1995; Bankaitis and Keith, 1995). The auditory brainstem evoked response (ABR) is an electrophysiological measure of nerve volleys and summated synaptic activity recorded from the scalp following presentation of a click stimulus. Rosenhall et al. (1989) investigated ABRs in HIV positive patients, who were free from opportunistic infection and had no symptoms that 
would suggest altered neurophysiology. They found that $38 \%$ of their subjects had increases in the I-V interpeak latency (IPL) difference. More recently, in an effort to demonstrate that the severity of abnormal neurophysiology may be related to the stage of HIV disease, Hausler et al (1991) recorded ABRs from both asymptomatic (CDC stage II and III) and symptomatic (CDC IV) HIV positive subjects. They found increased wave I-V interpeak latencies in $57 \%(n=14)$ of symptomatic patients compared to $45 \%(n=14)$ of symptomatic patients compared to $45 \%(n=29)$ of otherwise healthy HIV positive patients.

Animal models of HIV disease allow control over the time and method of inoculation as well as viral and host factors. Another important advantage is that the time course of disease can be greatly compressed. Currently, several promising animal models of HIV disease are available including feline immunodeficiency virus (FIV) infection in the cat and simian immunodeficiency virus (SIV) infection in the rhesus macaque (Vitkovic et al, 1995). Both FIV and SIV cause diseases similar in symptomology to HIV infection in humans (Lackner et al, 1991). Neurophysiological abnormalities have been reported in association with both FIV infection in the cat and SIV infection in the monkey (Podell et al, 1993; Phillips et al, 1994; Henriksen et al, 1995; Prospéro-García et al, 1996). For example, Henricksen et al (1995) and Podell et al (1993) reported that the latencies of peaks III and IV of the ABR increase in cats following inoculation with the Maryland strain of FIV. In the monkey, Prospéro-García et al. (1996) recently showed that the latencies of peaks IV and V and interpeak latencies (IPLs) I-IV and I$\mathrm{V}$ increase following infection with a microgliapassaged strain of SIV $_{\text {mac }} 251$.

Narayan and colleagues have demonstrated that two neurovirulent strains of $\operatorname{SIV}_{\text {mac }}$ (R71 and 17E), derived from bone marrow-passaged virus, enter the brain early following inoculation (Sharma et al, 1992; Joag et al, 1994a, b). However, it remains unclear whether there are any functional conse- quences of brain infection with these strains of SIV $_{\text {mac. }}$. As part of a larger comprehensive effort to characterize the behavioral and neurophysiological consequences of neurovirulent SIV infection in rhesus macaques, we recorded ABRs from nine macaques infected with neurovirulent SIV $_{\text {mac }}$ R71/ $17 \mathrm{E}$ both before infection and at regular intervals (every 4 to 6 weeks) following inoculation. Results presented in this paper show increases in peak times (II-V) as well as interpeak intervals I-V and III $-\mathrm{V}$.

\section{Results}

Morbidity and mortality

Seven of nine inoculated animals developed simian AIDS within 4 months and were euthanized. The mean post-inoculation survival time of these seven monkeys was 9.4 weeks (range 6-14 weeks, Table 1). All of these animals were considered to have rapidly progressing disease. Prior to euthanasia they showed signs of wasting, though caloric intake was strictly monitored and maintained, as well as diarrhea, tremor, ataxia, weakness, dermatitis, gingivitis and loss of appetite. Both of the remaining two inoculated animals became productively infected and were euthanized following 25 (AQ15) and 20 months (AQ94) post-inoculation. Both animals exhibited wasting but were otherwise free of overt clinical signs except for the presence of widely disseminated skin tumors in AQ15 and liver disease in AQ94.

\section{Latency changes}

ABRs were recorded ipsilaterally in association with monaural clicks delivered to the right ear. Peaks I-V were visually identified and latencies and amplitudes were computer measured using a windowing device. Data were automatically transferred to a spreadsheet and plotted. Interpeak latencies (IPLs) were also calculated and plotted. Table 2 is a summary of the mean pre- and postinoculation peak and interpeak latencies for all nine

Table 1 SIV $_{\max }$ R71/17E Cohort

\begin{tabular}{|c|c|c|c|c|c|c|c|c|}
\hline & Monkey & $\begin{array}{c}\text { Disease } \\
\text { progression }\end{array}$ & $\begin{array}{c}\text { Necropsy } \\
\text { date }\end{array}$ & $\begin{array}{c}\text { Survival time } \\
\text { (wks) }\end{array}$ & $\begin{array}{l}\text { Onset change } \\
\text { (wks p.i.) }\end{array}$ & $\begin{array}{c}\text { Total \# EP } \\
\text { sessions }\end{array}$ & $\begin{array}{c}\text { \# Control } \\
\text { sessions }\end{array}$ & $\begin{array}{c}\text { \# Post-inoc } \\
\text { sessions }\end{array}$ \\
\hline \multirow[t]{5}{*}{ Group A } & AQ 69 & Rapid & $3 / 16 / 96$ & 9 & 6 & 3 & 2 & 1 \\
\hline & $\mathrm{AQ} 70$ & Rapid & 4/18/96 & 14 & 6 & 5 & 2 & 3 \\
\hline & $\mathrm{AQ} 43$ & Rapid & $2 / 23 / 96$ & 6 & 6 & 3 & 2 & 1 \\
\hline & $\mathrm{AQ} 12$ & Rapid & $4 / 16 / 96$ & 14 & 6 & 5 & 2 & 3 \\
\hline & AQ 15 & Slow & $2 / 10 / 98$ & 109 & 0 & 16 & 2 & 14 \\
\hline \multirow[t]{4}{*}{ Group B } & AQ 94 & Slow & $3 / 4 / 98$ & 87 & 0 & 16 & 7 & 9 \\
\hline & $\mathrm{AQ} 47$ & Rapid & $8 / 27 / 96$ & 8 & 7 & 9 & 7 & 2 \\
\hline & AQ 38 & Rapid & $8 / 23 / 96$ & 7 & 7 & 9 & 7 & 2 \\
\hline & $\mathrm{AQ} 20$ & Rapid & $8 / 26 / 96$ & 8 & 0 & 8 & 7 & 1 \\
\hline
\end{tabular}

Five monkeys (Group A) were inoculated in 1/12/96. Four age and sex matched monkeys initially served as controls (Group B). Group B animals were inoculated on 7/3/96 after a period of control data was collected. 
monkeys. A consistent latency increase was observed in all of the ABR peaks; however, only peaks II-V achieved statistical significance. Significant increases in the interpeak latencies were observed for intervals $\mathrm{I}-\mathrm{V}$ and III $-\mathrm{V}$.

Table 3 gives the peak and interpeak latency increases that were significant in individual monkeys. Only two of the seven monkeys with rapidly progressing disease (AQ38 and AQ20) failed to show an increase in peak latency or IPL exceeding two standard deviations of control. The remaining five rapidly progressing monkeys showed increases in most peak and interpeak latencies, although not all increases exceeded the two standard deviation criterion. Neither of the two slow progressing animals, AQ15 and AQ94, showed increases in ABR latency.

Latency increases occurred within seven weeks of infection for all of the animals with rapidly progressing disease except one (AQ20) (Table 1). However, in most cases the increases only achieved two standard deviations of control when the animals were in the final stages of disease. Even at the time of necropsy, AQ20 failed to show an increase in ABR latency. For animals AQ70 and AQ12, the observed latency changes increased in severity following their initial onset.
Figure 1 shows examples of ABRs from an infected monkey (AQ43) and a control monkey (AQ47). Note the highly reproducible nature of the ABR in the control monkey recorded over a period of several months. Comparing the pre- and postinoculation records in the infected monkey reveals latency shifts in all of the ABR peaks. These delays are more easily recognized when the pre- and postinoculation records are superimposed (Figure 2).

No significant changes in ABR threshold over the course of disease progression were detected in any of the monkeys. The mean threshold both preinoculation and post-inoculation was $4.4 \pm 1.3$ (s.d.) $\mathrm{dB}$ for all of the animals except AQ94.

\section{Virology}

Following inoculation, virological data were collected every week for the first month, every 2 weeks for the second month and every 4 weeks thereafter. In addition to complete blood chemistry, CD4 and CD8 counts were also measured along with viral protein levels (p27) from the plasma (Table 3). P27 levels served as the primary indicator of productive viral infection while the presence of virus was confirmed by infectious cell assays (data not shown). The mean number of CD4 cells/ $\mu$ l of blood for all animals prior to inoculation was $1408 \pm 1126$

Table 2 Staistical analysis of ABR latency changes

\begin{tabular}{|c|c|c|c|c|c|c|c|}
\hline \multirow[b]{2}{*}{ Parameter } & \multirow{2}{*}{\multicolumn{2}{|c|}{$\begin{array}{c}\text { Mean value (ms } \\
\text { Post- } \\
\text { infection }\end{array}$}} & \multicolumn{3}{|c|}{ Summary of ABR Latency Changes: } & \multicolumn{2}{|c|}{ Variance components } \\
\hline & & & Difference & $P$-value & $\begin{array}{l}\text { Between } \\
\text { monkey }\end{array}$ & $\begin{array}{l}\text { Within } \\
\text { monkey }\end{array}$ & $\begin{array}{c}\text { Std. error of the } \\
\text { difference }\end{array}$ \\
\hline Peak 1 & 1.257 & 1.306 & 0.052 & 0.28 & 0.083 & 0.018 & 0.047 \\
\hline Peak 2 & 1.926 & 2.020 & 0.094 & 0.028 & 0.012 & 0.014 & 0.042 \\
\hline Peak 3 & 2.851 & 2.980 & 0.129 & 0.0016 & 0.0022 & 0.013 & 0.038 \\
\hline Peak 4 & 3.867 & 4.041 & 0.174 & 0.034 & 0.025 & 0.055 & 0.080 \\
\hline Peak 5 & 5.198 & 5.552 & 0.354 & 0.004 & 0.015 & 0.121 & 0.12 \\
\hline Peaks 1 to 3 & 1.599 & 1.662 & 0.063 & 0.22 & 0.087 & 0.020 & 0.05 \\
\hline Peaks 3 to 5 & 2.351 & 2.569 & 0.218 & 0.048 & 0.005 & 0.105 & 0.11 \\
\hline Peaks 1 to 5 & 3.923 & 4.281 & 0.358 & 0.0076 & 0.066 & 0.135 & 0.13 \\
\hline
\end{tabular}

Table 3 Summary of peak and interpeak latency changes

\begin{tabular}{|c|c|c|c|c|c|c|c|c|c|c|c|}
\hline \multirow[b]{2}{*}{ Monkey } & \multirow[b]{2}{*}{$I$} & \multirow[b]{2}{*}{$I I$} & \multirow[b]{2}{*}{$\begin{array}{l}\text { Peaks } \\
\text { III }\end{array}$} & \multirow[b]{2}{*}{$I V$} & \multirow[b]{2}{*}{$V$} & \multirow[b]{2}{*}{$I-V$} & \multirow[b]{2}{*}{$\begin{array}{c}I P L S \\
I I I-V\end{array}$} & \multirow[b]{2}{*}{$I-I I I$} & \multirow[b]{2}{*}{$\begin{array}{c}\text { Neuro } \\
\text { Pathology }\end{array}$} & \multicolumn{2}{|c|}{ Virology } \\
\hline & & & & & & & & & & $\begin{array}{c}p 27 \\
(p g / m l)\end{array}$ & $\begin{array}{c}C D 4^{*} \\
(\text { cells/ } \mu \mathrm{l})\end{array}$ \\
\hline AQ 69 & 0 & $\uparrow$ & $\uparrow$ & $\uparrow$ & 0 & 0 & $\uparrow$ & 0 & Mild & 1288 & 965 \\
\hline AQ 70 & $\uparrow$ & $\uparrow$ & $\uparrow$ & $\uparrow$ & $\uparrow$ & $\uparrow$ & $\uparrow$ & $\uparrow$ & Moderate & 2321 & 2356 \\
\hline AQ 12 & 0 & $\uparrow$ & $\uparrow$ & $\uparrow$ & 0 & $\uparrow$ & 0 & $\uparrow$ & Severe & 3830 & 658 \\
\hline AQ 43 & 0 & $\uparrow$ & $\uparrow$ & 0 & $\uparrow$ & $\uparrow$ & $\uparrow$ & $\uparrow$ & Moderate & 4902 & 518 \\
\hline AQ 47 & 0 & $\uparrow$ & $\uparrow$ & $\uparrow$ & $\uparrow$ & $\uparrow$ & $\uparrow$ & $\uparrow$ & $\operatorname{Mild}^{* *}$ & 5645 & 1540 \\
\hline AQ 38 & 0 & 0 & 0 & 0 & 0 & 0 & 0 & 0 & Moderate & 5238 & 1187 \\
\hline AQ 20 & 0 & 0 & 0 & 0 & 0 & 0 & 0 & 0 & Mild & 5688 & 51 \\
\hline AQ 15 & 0 & 0 & 0 & 0 & 0 & 0 & 0 & 0 & Mild & 225 & 177 \\
\hline AQ 94 & 0 & 0 & 0 & 0 & 0 & 0 & 0 & 0 & Mild & $0 \dagger$ & 119 \\
\hline
\end{tabular}

$\uparrow$, Increase $\geqslant 2$ std. deviations. 0 , No change. ${ }^{*}$ The normal range for CD4 cells is $1000-2000 / \mu l .{ }^{*}$ Based on H\&E staining. $\uparrow \mathrm{AQ} 94$ had viral p27 levels as high as 332 at earlier time points, confirming productive infection. 
CONTROL

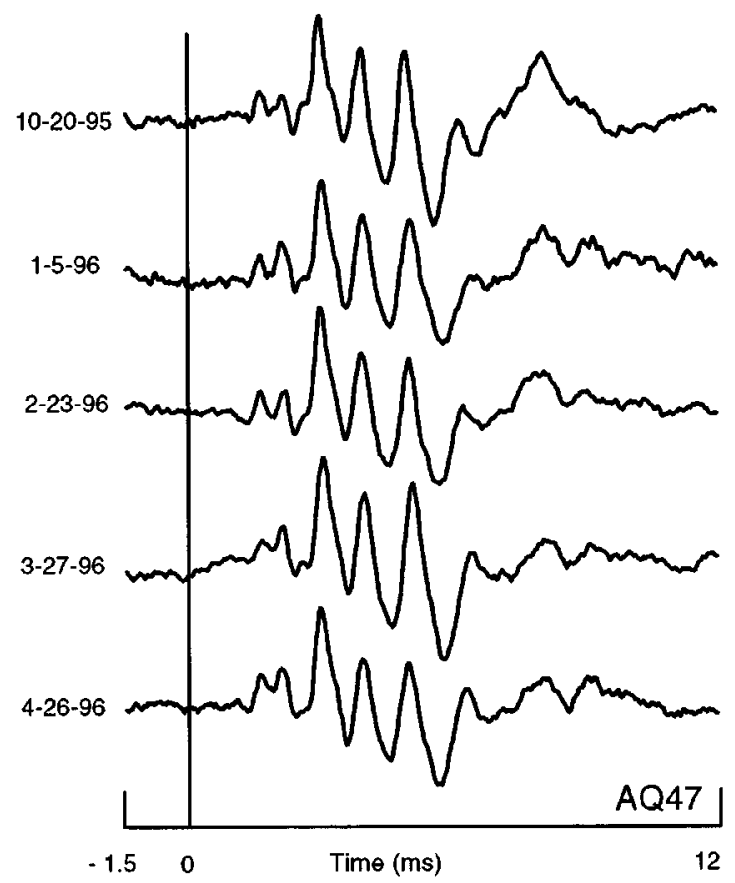

SIV INFECTED

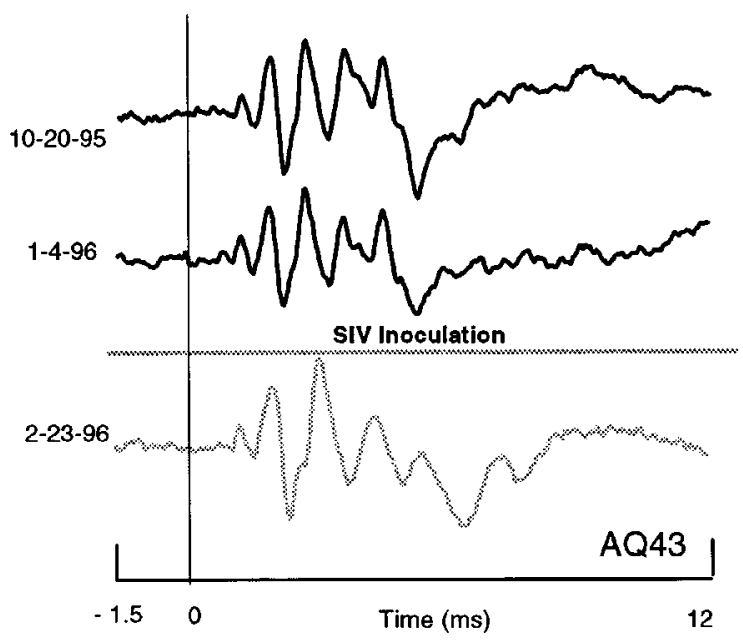

Figure 1 Serial ABRs recorded from a control (AQ47) and SIV-infected (AQ43) monkey at similar time points during the course of the study. Note the consistency of the ABR waveform from AQ47 over a 7 month period. Also note the increase in peak latencies of AQ43's ABR following SIV infection.

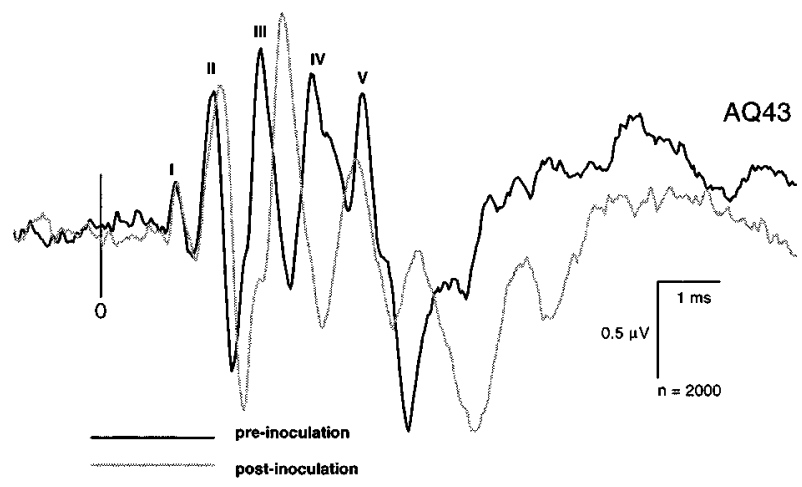

Figure 2 Superimposed ABR records for AQ43 obtained during the control period (10-20-95) and following SIV inoculation (223-96). The latencies of peaks II-V, are clearly increased. Latencies were measured from the onset of the trigger stimulus.

(s.d.). Following inoculation, mean CD4 levels dropped, though only to $841 \pm 761.82$ (s.d.). The appearance of p27 in blood occurred within three weeks of inoculation for all animals. At 3 weeks post-inoculation, the mean p27 level for all animals was $3212 \pm 1294 \mathrm{pg} / \mathrm{ml}$ (s.d.). The mean p27 level at the time of euthanasia for the seven monkeys with rapidly progressing disease was $4130 \pm 1731 \mathrm{pg} / \mathrm{ml}$ (s.d.). In contrast, the two slow progressors, AQ15 and AQ94 developed only transient viremia and rapidly gained control of virus replication showing transient CD4 cell loss and only limited evidence of virus replication. The slow progressing disease exhibited by these two animals is reminiscent of the slowly or non-progressive type of infection observed in some people infected with HIV. At the time of euthanasia, AQ15 had a CD4 cell count of 177 and a viral load of 225; AQ94 had a CD4 cell count of 119 and a viral load of 0 . Although, p27 levels in AQ94 were undetectable at the time of euthanasia, a p27 concentration of $332 \mathrm{pg} / \mathrm{ml}$ was detected at an early time point in disease progression.

\section{Histopathology}

Evidence of typical retroviral induced CNS neuropathology was observed in six of eight animals from which tissue was available (Tables 3 and 4). A rigorous analysis of the brainstem auditory structures was done using sections stained with thionin (Table 4). Broadly similar results were obtained from a more limited analysis based on hematoxylin/ eosin (H\&E) stained sections. AQ69 did not exhibit typical SIV neuropathology, but rather encephalitic foci and necrotic lesions consistent with cytomegalovirus infection. AQ47's brain was not examined 
using the thionin stain due to tissue unavailability, but instead was examined using the standard H\&E protocol. The thionin-stained auditory structures that were examined included the cochlear nuclear complex, the lateral lemniscus, the olivary complex, the inferior colliculus, and the medial geniculate body. Pathological findings included perivascular mononuclear infiltrates (PVCs), microglial nodules (MGNs) and multinucleate giant cell (GCs) reactions. The severity of the disease (based on the extent of dissemination, intensities, and type of inflammatory response) varied from animal to animal (Tables 3 and 4). All five animals that exhibited ABR IPL increases had evidence of lesions in the brainstem auditory pathway. However, the severity of the neuropathology did not always correlate with the severity of the ABR latency increases. For example, AQ47 showed only mild pathology despite the fact that this monkey exhibited marked increases in ABR peaks I-V and all interpeak intervals. The reverse also occurred and was more common. For example, AQ38 exhibited moderate neuropathology but had no significant increases in ABR peak or interpeak latencies. It is interesting to note that neither of the two animals with slow progressing disease

Table 4 Auditory pathway pathology

\begin{tabular}{lccccc}
\hline & & & & & \multicolumn{2}{c}{ Med } \\
Monkey & $\begin{array}{c}\text { Cochlear } \\
\text { complex }\end{array}$ & $\begin{array}{c}\text { Lat. } \\
\text { leminiscus }\end{array}$ & $\begin{array}{c}\text { Olivary } \\
\text { complex }\end{array}$ & $\begin{array}{c}\text { Inferior } \\
\text { colliculus }\end{array}$ & $\begin{array}{c}\text { geniculate } \\
\text { body }\end{array}$ \\
\hline AQ 69 & + & + & + & + & + \\
AQ 70 & N/A & + & ++ & ++ & + \\
AQ 38 & + & + & ++ & ++ & ++ \\
AQ 43 & + & + & ++ & + & + \\
AQ 15 & + & 0 & + & + & 0 \\
AQ 94 & 0 & 0 & + & 0 & + \\
AQ 12 & ++ & +++ & ++ & ++ & ++ \\
AQ 20 & 0 & + & + & + & + \\
\hline
\end{tabular}

Note: AQ 47 was unavailable for thionin analysis. N/A: not available. $0=$ no pathology; + , mild; ++ , moderate; +++, severe.

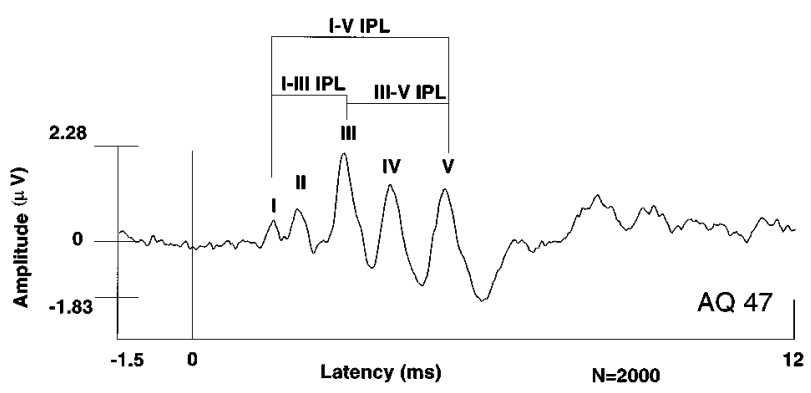

Figure 3 Typical macaque auditory brainstem response (ABR) consisting of five peaks $(\mathrm{I}-\mathrm{V})$. The latency of individual peaks as well as interpeak latencies (I-III, III-V and I-V), were measured from the trigger stimulus and were studied as a measure of the functional integrity of the brainstem auditory system. showed typical retroviral neuropathology. AQ15 showed only mild, focal meningo-encephalitis and AQ94 had no remarkable neuropathological changes.

\section{Discussion}

The overall objective of this study was to further characterize the neurovirulent SIV $_{\text {mac }}$ R71/17E model of neuro-AIDS. The auditory brainstem response provides a physiological measure of auditory pathway impairment and CNS involvement associated with SIV infection. Our results demonstrate ABR peak delays, and interpeak interval increases in SIV infected monkeys similar to those that have been reported in HIV infected humans.

Because of its reproducibility and robustness, the ABR has been routinely used as a diagnostic test for demyelinating diseases of the CNS such as multiple sclerosis as well as a test for conductive and sensory-neuronal hearing loss in infants and other difficult to test patients (Schwartz and Grant, 1985). The proximal and distal portions of cranial nerve VIII are thought to be the neural generators of ABR peaks I and II, respectively (Donohoe, 1988). The superior olivary nucleus is thought to be the generator of peak III, while peak IV is attributed to the lateral lemniscus. Peak V is believed to originate from the inferior colliculus. By examining the IPLs of subjects, it is possible to draw conclusions pertaining to the etiology and location of possible pathology.

ABR data presented in this paper substantiates functional impairment of the brainstem auditory relay system in SIV infected monkeys. The increases in latency of peaks III-V suggest lesions or demyelination in the upper brainstem. In all cases lesions in these locations have been confirmed by pathology. We also observed small increases in the latency of peak II suggesting possible demyelination or conduction inpairment in cranial nerve VIII, which was unavailable for pathological examination.

It should be noted that while retroviral pathology and functional deficits are often associated, our study of ABR pathophysiology revealed some instances in which the two did not match. All monkeys with ABR abnormalities showed neuropathology, although in some cases the severity of pathology was mild (AQ47). This is consistent with reports from human studies in which some subjects with HIV dementia had no associated neuropathology (Navia et al, 1986b; Glass et al, 1993). The reverse also occurred; AQ38 had moderate neuropathology but no significant ABR pathophysiology. These findings, taken as a whole, suggest that functional injury and eventual neuronal death may be the most important pathologic event resulting from retroviral infection and this may 
occur with little or no classic neuropathology, ie. inflammatory lesions. Such functional injury might take the form of abnormal firing activity, conduction block, or impairment in synaptic transmission.

Animal investigations pertaining to the pathophysiology associated with lentivirus infection have, in the past, focused on the FIV model. Podell et al (1993); Phillips et al (1994); Henriksen et al (1995) all found increases in ABR latency following FIV infection. More recently, Prospéro-García et al (1996), reported increases in latency of ABR peaks in SIV infected macaques. Our results complement theirs in showing preferential effects on IPL III-V as opposed to IPL I-III (Table 2). This suggests that central auditory pathway function is more severely affected than peripheral structures by SIV infection. Retroviral damage to the CNS most clearly manifests when disease progresses to end-stage AIDS, and our study of ABRs in SIV infected macaques have confirmed this. Although latency shifts began early in some cases, the most dramatic changes were associated with end-stage disease.

In summary, our results have demonstrated clear ABR peak latency and IPL shifts associated with $\mathrm{SIV}_{\text {mac }} \mathrm{R} 71 / 17 \mathrm{E}$ infection in the monkey. These shifts reflect underlying dysfunction in the auditory pathway, particularly more central components of the pathway, possibly associated with demyelination and/or other pathophysiological processes. In conclusion, our results lend further support to the validity and potential usefulness of the $\operatorname{SIV}_{\mathrm{mac}}$ model of neuro-AIDS. We believe this model has great potential for in-vivo studies of pathophysiology and therapeutic interventions.

\section{Materials and Methods}

\section{Subjects}

Nine male rhesus macaques (Macaca mulatta) of Indian origin and free of the Simian Retrovirus-D and Herpes B virus served as subjects for recording evoked potentials. The monkeys were also trained to perform a behavioral test battery that included measures of reaction time, working memory and decision making ability. All nine monkeys were inoculated via bone marrow injection of the femur with two neurovirulent strains of $\operatorname{SIV}_{\text {mac }}$ (R71 and 17E). Each animal received $0.5 \mathrm{ml}$ of each infection cell-free homogenate for a total of volume of $1 \mathrm{ml}$ that contained approximately 1000 TCID. Initially, five monkeys were inoculated (Group A) and four monkeys served as controls (Group B). These four monkeys were then also inoculated six months after the Group A monkeys (Table 1).

Animals were individually housed following Animal Biosafety Level 2 criteria in an AAALAC accredited facility. Daily temperature and health observations were noted and home cage behavior was recorded and rigorously scored weekly. Prior to inoculation, a baseline MRI was obtained along with blood and cerebrospinal fluid (CSF) samples. Blood and CSF samples were then collected from all animals once a week for the first month after inoculation, every 2 weeks for the second month, and thereafter, at 4 week intervals. Within 14 weeks of inoculation, 6 of 9 animals developed end-stage simian AIDS and were euthanized. One monkey, AQ69, died while recovering from isoflurane anesthesia for a MRI procedure. This monkey showed signs of wasting but had not entered endstage disease. Two animals (AQ15 and AQ94) were productively infected but had slowly-progressing disease and were euthanized at 25 and 20 months due to systemic complications including wasting, liver disease and skin tumors. All procedures performed on these animals were approved by the University of Kansas Medical Center Animal Care and Use Committee and complied with the Guide for the Care and Use of Laboratory Animals established by the U.S. Department of Health and Human Services, National Institutes of Health.

The decision to euthanize was based on progression to any one of the following conditions: (1) wasting that exceeded $20 \%$ loss of original body weight, (2) failure to maintain an upright sitting posture, (3) severe loss of appetite, or (4) diarrhea unresponsive to treatment.

\section{Data collection}

At 1 month prior to inoculation and every 4-6 weeks thereafter, daily behavioral training/testing was suspended for 1-2 days so that ABRs could be recorded. Following each evoked potential (EP) session, all of the animals were allowed to recover for 2 days from any effects of anesthesia and then were returned to the behavioral test battery. They showed no lingering behavioral effects from anesthesia or evoked potential testing. Following an initial injection of atropine $(0.05 \mathrm{mg} / \mathrm{kg})$ and ketamine $(10 \mathrm{mg} / \mathrm{kg})$, anesthesia was maintained by subsequent doses of ketamine $(10 \mathrm{mg} / \mathrm{kg})$ at $20-$ 30 min intervals. ABR latency did not vary over our cycle of ketamine administration.

Using an infrared tympanic thermometer, temperature was measured on all monkeys at the beginning and end of each EP session. Body temperature was sustained by surrounding the animal with heated saline bottles during recording. Both ears were debrided before recording sessions.

ABRs were recorded in a sound attenuating chamber using Grass ${ }^{\mathrm{TM}}$ AC preamplifiers (Model P511) with filter settings of $100 \mathrm{~Hz}-3 \mathrm{kHz}$ and a gain of $200 \mathrm{~K}$. A Grass ${ }^{\mathrm{TM}}$ auditory click stimulator (Model S10CTCMA) was used to generate $100 \mu \mathrm{s}$ square wave pulses at a frequency of $11.5 \mathrm{~Hz}$. The square wave pulse was used to drive an E-ARTone $^{\mathrm{TM}}$ tubephone (Model 3A; Cabot Safety Corporation, Indianapolis, IN) producing a click with a sound pressure level (SPL) of $88 \mathrm{~dB}$. The tubephone was fitted to the right external ear canal 
with a foam gasket. A total of 2000 stimulus repetitions was averaged and recorded using a custom Windows ${ }^{\mathrm{TM}}$ compatible software program (Neural Averager, Larry Shupe, Seattle, Washington) written for Cambridge Electronic Design (CED 1401 plus) data acquisition hardware. ABR thresholds were determined at a gain of $200 \mathrm{~K}$ while adjusting the SPL level of the stimulus. Peak V of the ABR typically had the lowest threshold. Each animal's ABR threshold was measured during each EP session. Thresholds were measured to within $\pm 2 \mathrm{~dB}$.

ABRs were recorded using the international 1020 electrode configuration and subdermal platinum needle electrodes (Grass ${ }^{\mathrm{TM}}$ model F-E2). CZ served as the active electrode and the right mastoid as the reference electrode. The right shoulder provided a noncephalic ground.

\section{Off-line analysis}

Averages for all EP sessions for a given monkey were combined in a single computer file for off-line measurement. The resulting summary file for each monkey served as a template for visual identification of peaks. Peak times and amplitudes were computer measured. Five peaks were identified (IV) and three interpeak latencies (IPLs) were measured: I-III, III-V and I-V (see Figure 3). These peaks can easily be identified in serial records covering more than 5 months including the control period (Figure 1). The waveform and relative size of peaks was very reproducible representing a signature for each monkey.

\section{Statistical methods}

Mean differences in peak times and interpeak time intervals between pre- and post-inoculation periods were examined with a linear mixed model. This model is analogous to a repeated measures ANOVA, but it is amenable to the variability in numbers of pre- and post-inoculation observations for different animals. The mixed model approach allowed us to gain power from the individual observations on each animals while obtaining correct inference by controlling for the correlation in the multiple observations within a given monkey. $P \leqslant 0.05$ was considered significant.

Graphical procedures were used to examine latency changes in individual animals. The procedure used is analogous to Shewert control charts often used in industrial process control (Box et al, 1978). To generate the estimates of within monkey variability needed to construct the control charts, a variance components analysis was applied to the pre-inoculation observations from the nine animals to partition the different peak times and interpeak time intervals into between-animal and withinanimal components. Control charts for each animal were then constructed using the mean pre-inoculation peak latencies or interpeak times for that animal and the average within-monkey standard deviation obtained from the variance components analysis. Plots of the post-inoculation peak times and intervals were then compared to the expected range (mean \pm 2 standard deviations) to assess whether SIV infection was indicative of 'loss of control' of the system.

\section{Histology}

At necropsy, the animals were tranquilized with ketamine and anesthetized with pentobarbital $(10 \mathrm{mg} / \mathrm{kg})$ given intravenously until a surgical plane anesthesia was achieved. They were then exsanguinated from the descending aorta and perfused transcardially with two liters of normal saline followed by two liters of $10 \%$ neutral buffered formalin. AQ47 and AQ43 were not perfused with formalin so that fresh tissue samples could be collected for virological and molecular analyses. The brains were removed, fixed in $10 \%$ formalin for 3-4 days and hemisectioned. The right hemisphere was dissected in the coronal plane and representative areas from 12-14 regions of the hemisphere and brainstem were embedded in paraffin, sectioned at $5 \mu \mathrm{m}$ and stained with $\mathrm{H} \& \mathrm{E}$ for histopathological analysis. In the case of macaques, AQ43 and AQ47, the freshly removed brain was dissected in a biosafety hood and 12-14 regions of the central hemisphere and brainstem were snap-frozen and stored at $-70^{\circ} \mathrm{C}$ for DNA analysis. Portions of the CNS tissue were also saved for viral isolation. The remaining portions of the right hemisphere were immersion fixed in $10 \%$ formalin and dissected in the same manner as the other animals. The left hemisphere was fixed in $10 \%$ formalin, blocked in the standard coronal plane and cryoprotected in $30 \%$ sucrose in $0.1 \mathrm{M}$ phosphate buffer for stereological and immunohistochemical analysis. A sliding frozen microtome was used to section ( $50 \mu \mathrm{m}$ ) through a $38 \mathrm{~mm}$ block of tissue that included both the motor and sensory cortices as well as the entire basal ganglia and a large portion of the brainstem through the inferior olive. A 1/10 series of these sections was then mounted and stained with $1 \%$ thionin. The left hemisphere of AQ47 was not sectioned or stained with thionin due to its use in virological studies. AQ69, though not included in the $\mathrm{H} \& \mathrm{E}$ analysis, was sectioned and stained with thionin.

\section{Auditory pathway analysis}

Sections through the cochlear nuclear complex, lateral lemniscus, the olivary complex, the inferior colliculus, and the medial geniculate body were identified using the Snider and Lee (1960)

Stereotaxic Atlas of Macaqua mulutta A $40 \times$ planapochromatic objective, NA.65 attached to a Nikon microscope was used to examine the thionin sections. The presence, location and the number of 
microglial nodules (MGNs), perivascular cuffine (PVCs), and existence of multinucleate giant cells (GCs) was noted for each animal. A 0 to +++ scoring system was developed to rank the severity of neuropathology in each animal. The mean number of PVCs, MGNs and GCs were calculated for each area examined; a score of 0 was given if no PVCs, MGNs or PVCs were observed; a + score was given if pathology was present and the number of foci were within 1 standard deviation of the mean calculated for all monkeys, ++ if the number of foci were between one and two standard deviations of the mean; those areas exceeding two standard deviations were given a score of +++ . Using this system a maximum score of 9 was possible, +++ for PVCs, +++ for MGNs, +++ for GCs for each area examined. To simplify the rating system, the following composite score assignments were made: $1-3,+$; 4$6,++; 7-9,+++$. The total number of +'s was then summed to derive an overall neuropathology score

\section{References}

Arendt G, Hefter H, Elsing C, Strohmeyer G and Freund HJ (1990). Motor dysfunction in HIV-infected patients without clinically detectable central-nervous deficit. $J$ Neurol 237: 362-368.

Arendt G, Hefter $\mathrm{H}$ et al (1992). Improvement of motor performance of HIV-positive patients under AZT therapy. Neurology 42: 891-896.

Bankaitis AE (1995). The effects of click rate on the auditory brain stem response (ABR) inpatients with varying degrees of HIV-infection: a pilot study. Ear Hear 16: $321-324$.

Bankaitis AE and Keith RW (1995). Audiological changes associated with HIV infection. Ear Nose Throat $J$ 74: $353-359$.

Birchall MA, Wight RG et al (1992). Auditory function in patients infected with the human immunodeficiency virus. Clin Otalaryngol 17: 117-121.

Bornstein RA, Nasrallah HA, Para MF, Whitacre CC, Rosenberger P, Fass RJ and Rice R (1992) Neuropsychological performance in asymptomatic HIV infection. J Neuropsychiatry and Clin Neurosci 4: 386394.

Box GEP, Hunter WG, Hunter JS (1978). Statistics for Experiments. J. Wiley And Sons. New York: pp 556563.

Butters N, Grant I, Haxby J, Judd LL, Martin A, McClelland J, Pequegnat W, Schacter D and Stover E (1990). Assessment of AIDS-related cognitive changes: recommendations of the NIMH Workshop on Neuropsychological Assessment Approaches. J Clin Exp Neuropsychol 12: 963-978.

Clifford DB, Jacoby RG et al (1990). Neuropsychometric performance of asymptomatic HIV-infected subjects. Aids 4: 767-774.

Donohoe CD (1988). Application of the Brainstem Auditory Evoked Response in Clinical Neurological Practice. IN: Clinical Atlas of Auditory Evoked Potentials. J.H.A.D. Owen, C.D. Orlando, FI, Grune and Stratton, Inc.: Chap 3 pp 29-43. for each monkey for the auditory pathway; total maximum score possible was 15 (five areas with +++ ). Each animal's composite neuropathology score appears in Table 3 (none: 0; mild: 1-5; moderate: 6-10; severe; $11-15$ ).

\section{Acknowledgements}

The authors wish to thank Dr John Ferraro for excellent suggestions regarding ABR measurement and analysis, and Randell Lininger, Jill BrandomHarris and Jennifer English for their expert technical contributions. We also thank Jim Rengel and Ted Gleason for assistance with electronics and Dr David Pinson, for assistance with the necropsies. This research was supported by NIH grants NS32203 and HD02528.

Egan V (1992). Neuropsychological aspects of HIV infection. Aids Care 4: 3-10.

Elder GA et al (1988). AIDS and neurological disorders: an overview. Ann Neurology 23(Supplement): S4-6.

Glass JD, Weselingh SL, Seines OA, and McArthur JC (1993). Clinical-neuropathological correlation in HIVassociated dementia. Neurology 43: 2230-2237.

Hausler R, Vibert D et al (1991). Neuro-otological manifestations in different stages of HIV infection. Acta Otolaryngol Suppl Stockh 481: 515-521.

Henriksen SJ, Prospéro-García O et al (1995). Feline immunodeficiency virus as a model for study of lentivirus infection of the central nervous system. Curr Top Microbiol Immunol 202: 167-186.

Janssen RS, Saykin AJ et al (1989). Neurological and neuropsychological manifestations of HIV-1 infection: association with AIDS-related complex but not asymptomatic HIV-1 infection. Ann Neurol 26: $592-$ 600.

Joag SV, Adams RJ, Foresman L, Galbreath D, Zink MC, Pinson DM, McClure H, and Narayan O (1994a). Early activation of PBMC and appearance of antiviral CD8+ cells influence the prognosis of SIV-induced disease in rhesus macaques. J Med Primatol 23: 108-116.

Joag SV, Adams RJ, Pinson DM, McClure H and Narayan O (1994b). Intracerbral infusion of TNF-a and IL-6 failed to activate latent SIV infection in the brain of macaques inoculated with macrophage-tropic neuroadapted SIV mac. J Leukoc Biol 56: 353-357.

Karlsen NR, Reinvang I and Froland SS (1992a). A follow-up study of neuropsychological function in asymptomatic HIV-infected patients. Acta Neurol Scand 87: 83-87.

Karlsen NR, Reinvang I and Froland SS (1992b). Slowed reaction time in asymptomatic HIV-positive patients. Acta Neurol Scand 86: 242-246.

Krikorian R and Wrobel AJ (1991) Cognitive impairment in HIV infection. Aids 5: 1501-1507. 
Lackner AA, Dandekar S et al (1991). Neurobiology of simian and feline immunodeficiency virus infections. Brain Pathol. 1: 201-212.

Martin EM, Sorensen DJ, et al (1992a). Decision-making speed in HIV-1 infection: a preliminary report. Aids 6: 109-113.

Martin EM, Sorensen DJ et al (1992b). Spatial attention in HIV-1 infection: a preliminary report. J Neuropsychiatry Clin Neurosci 4: 288-293.

Martin EM, San Giovanni D, Sorensen DJ, Robertson LC and Edelstein HE (1991). Performance of patients with early HIV-1 infection on the stroop task: comparison of standard and reaction time-based measures. J Clin Exp Neuropsychol 13: 73-74.

McAllister RH, Herns MH et al (1992). Neurological and neuropsychological performance in HIV seropositive men without symptoms. J Neurol Neurosurg Psychiatry 55: $143-148$.

Navia BA and Price RW (1987). The acquired immunodeficiency syndrome dementia complex as the presenting or sole manifestation of human immunodeficiency virus infection. Arch Neurol 44: 65-69.

Navia BA, Jordan BD and Price RW (1986a). The AIDS dementia complex: I. Clinical features. Ann Neurol 19: $517-524$.

Navia BA, Cho E-S, Petito CK, Price RW (1986b). The AIDS dementia complex II. Neuropathology. Ann Neurol 19: $525-535$.

Pagano MA, Cahn PE et al (1992). Brain-stem auditory evoked potentials in human immunodeficiency virusseropositive patients with and without acquired immunodeficiency syndrome. Arch Neurol 49: 166169.

Perry S and Marotta RF (1987). AIDS dementia: a review of the literature. Alzheimer Dis Assoc Disord 1: $221-$ 235.

Perry S, Belsky BD et al (1989). Neuropsychological function in physically asymptomatic, HIV-seropositive men. J Neuropsychiatry Clin Neurosci 1: 296-302.

Phillips TR, Prospéro-García O et al (1994). Neurological abnormalities associated with feline immunodeficiency virus infection. J Gen Virol 75: 979-987.
Podell M, Oglesbee $M$ et al (1993). AIDS-associated encephalopathy with experimental feline immunodeficiency virus infection. J Acquir Immune Defic Syndr 6: $758-771$.

Price RW, Brew B, Sidtis JJ, Rosenblum J, Sheck AC, Cleary P (1988). The brain in AIDS: Central nervous system HIV-1 infection and AIDS dementia complex. Science 239: $586-592$.

Prospéro-García O, Gold LH, et al (1996). Microglia passaged simian immunodeficiency virus induces neurophysiological abnormalities in monkeys. Proc Natl Acad Sci USA 93: 14158-14163.

Riedel RR, Helmstaedter C, et al (1992). Early signs of cognitive deficits among human immunodeficiency virus-positive hemophiliacs. Acta Psychiatr Scand 85: $321-326$.

Rosenhall U, Hakansson C, et al (1989). Otoneurological abnormalities in asymptomatic HIV-seropositive patients. Acta Neurol Scand 79: 140-145.

Schwartz DM, Grant A (1985). Normative Aspects of the ABR. IN: The Auditory Brain Response. J. Jacobson, San Diego, CA., College Hill Press: Chap. 5, pp 6592.

Sharma DP, Zink MC, Anderson M, Adams R, Clements JE, Joag SV and Narayan O (1992). Derivation of neurotropic simian immunodeficiency virus from exclusively lymphocytetropic parental virus: pathogenesis of infection in macaques. J Virol 66: 35503556.

Sinforiani E, Mauri M et al (1991). Cognitive abnormalities and disease progression in a selected population of asymptomatic HIV-positive subjects. Aids 5: $1117-$ 1120.

Snider RS and Lee JC (1961). A Stereotaxic Atlas of the Monkey Brain: Macaca mulatta. The University of Chicago Press: Chicago.

Stern Y (1991). The impact of human immunodeficiency virus on cognitive function. Ann NY Acad Sci 640: $219-223$.

Vitkovic LE, Stover et al. (1995). Animal models recapitulate aspects of HIV/CNS disease. AIDS Res. Human Retroviruses 11: 753-759. 